\title{
La controversia por el vertedero de residuos en la Cordillera de los Andes. Un estudio de caso acerca de la responsabilidad internacional por daño transfronterizo
}

The Controversy over the Waste Dump in the Andean Mountains. A Case Study on International Liability for Transboundary Harm

\section{La controverse sur la décharge dans la Cordillère des Andes. Étude de cas sur la responsabilité internationale pour les dommages transfrontières}

Alexis Rodrigo Laborías*

\begin{abstract}
SUMARIO: I. Introducción. II. Apuntes acerca de la responsabilidad internacional ex delicto y sine delicto. III. Desarrollo de la controversia por el vertedero de residuos. IV. Solución del diferendo entre Argentina y Chile.

V. La controversia de la Cordillera a la luz de la responsabilidad internacional. VI. Conclusiones. VII. Bibliografía.
\end{abstract}

* Universidad Argentina de la Empresa, Argentina; ORCID ID: https://orcid.org/00000001-7414-0311,alaborias@uade.edu.ar. 
RESUMEN: El artículo analiza la controversia entre Argentina y Chile, con motivo del descubrimiento de un vertedero de residuos en la Cordillera de los Andes, originado por la actividad de una empresa minera chilena, que arrojaba el material en desuso en el territorio argentino. El conflicto es estudiado a la luz de las reglas en materia de responsabilidad internacional y teniendo en cuenta los mecanismos de solución de disputas a los que recurren los Estados ante un hecho de contaminación transfronteriza.

Palabras clave: responsabilidad internacional, hechos internacionalmente ilícitos, prevención del daño transfronterizo, responsabilidad por contaminación transfronteriza, solución pacífica de las controversias.

ABSTRACT: The article analyzes the controversy between Argentina and Chile, following the discovery of a waste dump in the Andes Mountains, originated in the activity of a Chilean mining company, which threw the disused material into Argentine territory. This dispute is studied in the light of the rules on international liability and taking into account the dispute resolution mechanisms to which States resort in the face of a transboundary harm event.

Key words: international liability, internationally wrongful acts, prevention of transboundary harm, liability for transboundary pollution, pacific settlement of disputes.

RÉSUMÉ: L'article analyse la controverse entre l'Argentine et le Chili à l'occasion de la découverte d'une décharge dans la Cordillère des Andes, née de l'activité d'une société minière chilienne, qui a jeté les matériaux en désuétude sur le territoire argentin. Le conflit est étudié à la lumière des règles de responsabilité internationale et des mécanismes de résolution des litiges auxquels les États ont recours en cas de pollution transfrontalière.

Mots-clés: responsabilité internationale, actes internationalement illicites, prévention des dommages transfrontières, responsabilité pour la pollution transfrontière, règlement pacifique des différends. 


\section{INTRODUCCIÓN}

Todo sistema jurídico entraña la búsqueda de una convivencia pacífica entre los sujetos, a través de la armonización de un conjunto de derechos y obligaciones. Este equilibrio no es sencillo de alcanzar y preservar, de ahí que el propio sistema disponga de ciertas herramientas para restablecer los desajustes que puedan producirse.

En este sentido, se prevé que los sujetos puedan infringir las reglas vigentes y, por este motivo, se estipulan una serie de consecuencias para dichos actos disvaliosos. Al aplicar una secuela a la violación de las normas se busca recuperar el equilibrio, reafirmando el alcance de los derechos y el tenor de las obligaciones. Asimismo, existen supuestos en los cuales, sin que se produzca una transgresión, el ejercicio legítimo de un derecho por parte de un sujeto puede tener una derivación perjudicial para terceros. La subsanación de esos efectos, previstos o imprevistos, no puede recaer en el sujeto damnificado, lo cual iría en detrimento del adecuado balance entre derechos y obligaciones. Es por este motivo que los sistemas jurídicos prevén que también debe ofrecer una compensación quien se beneficia con un derecho y que, al mismo tiempo, provoca un perjuicio indebido a un tercero.

Ambas situaciones son estudiadas bajo el concepto de responsabilidad, distinguiendo las hipótesis con terminología diversa: subjetiva u objetiva, por violación de normas o por consecuencias perjudiciales de actos lícitos, y ex delicto o sine delicto, respectivamente.

El derecho interno de cada Estado determina con exactitud de qué manera se atribuye la responsabilidad a un sujeto y cuál será su contenido, es decir, cuáles serán las nuevas obligaciones que se deben afrontar, ya sea por el incumplimiento de las obligaciones preexistentes o por los efectos perjudiciales surgidos del ejercicio legítimo de un derecho. Por cierto, en un sistema de derecho interno la coherencia se ve favorecida por su centralización, dado que una única autoridad estatal aprueba las normas de alcance general y obligatorio para todos los sujetos.

De manera opuesta, en la esfera internacional los derechos y obligaciones surgen a partir de la interacción de los sujetos, quienes buscan alcanzar objetivos comunes y mantener una convivencia armoniosa, a través del acuerdo de voluntades. Esta descentralización determina que muchas reglas del derecho internacional no presenten un carácter unívoco ni se encuentren 
adecuadamente organizadas, sino que se muestran más flexibles, con arreglo a las necesidades de los sujetos de este ordenamiento. Por supuesto, las normas que regulan la responsabilidad internacional, incluyendo la distinción entre ex delicto y sine delicto, no son ajenas a esta situación. ${ }^{1}$

En el ámbito de la Organización de las Naciones Unidas (ONU), la Comisión de Derecho Internacional (en adelante, "la Comisión" o "la CDI") se vio enfrascada en estos debates por largas décadas, al buscar el desarrollo progresivo y la codificación de las reglas sobre responsabilidad. Los propios miembros de la ONU fueron muy reticentes a avanzar en los caminos que proponía la CDI, y el análisis de la práctica estatal ofrece, en ocasiones, una imagen poco concluyente respecto de las reglas generales vigentes.

En este sentido, el objetivo de este trabajo será analizar una controversia acaecida entre Argentina y Chile, vinculada con un evento de contaminación transfronteriza. A tal efecto, se realizará una síntesis de los trabajos de la CDI en el campo de la responsabilidad internacional ex delicto y sine delicto. Posteriormente, se describirán los pormenores del conflicto bilateral y los acuerdos celebrados por ambos Estados para dirimir la disputa. Por último, a partir de esa información se esbozarán los motivos por los cuales ambos Estados negociaron y acordaron una solución pragmática, que no responde estrictamente a los estándares teóricos en materia de responsabilidad internacional.

\section{APUNTES ACERCA DE LA RESPONSABILIDAD}

INTERNACIONAL EX DELICTO Y SINE DELICTO

A partir de su experiencia como relator especial de la CDI, Barboza describió el estado de situación de las reglas en este campo:

1 De acuerdo con Rey Caro: "No es muy simple dar una noción o definición de responsabilidad en razón de las marcadas divergencias, a veces, sustanciales, sobre su función, sin perjuicio de que sea entendida como una regla o un conjunto de reglas que deben observarse. Existe entre los autores cierta coincidencia en destacar el carácter consuetudinario de las normas para hacer efectiva la responsabilidad. Sin embargo, tal concordancia desaparece a la hora de abordar la verdadera naturaleza de la o de las reglas en sí mismas”. Cfr. Rey Caro, Ernesto J., "La responsabilidad internacional. Reflexiones sobre su evolución”, en Rey Caro, Ernesto J. (dir.), Responsabilidad internacional, Córdoba, Academia Nacional de Derecho y Ciencias Sociales, 2009, p. 11. 
En Derecho, la responsabilidad por un daño puede nacer ex delicto, o sine delicto, según que el daño se haya o no producido por la violación de una obligación. La primera, tras vacilaciones y un proceso de codificación que duró alrededor de cuarenta años, es ya sin duda parte del Derecho Internacional general y encuentra expresión en los artículos incorporados a la Res. AGNU 56/83. La segunda no tiene carta de ciudadanía en el Derecho general, al menos para la doctrina más ortodoxa y para la opinio iuris de los Estados, pero campea en el Derecho particular en convenciones multilaterales que reglan actividades específicas. ${ }^{2}$

La Comisión realizó un prolongado y arduo esfuerzo para alcanzar el desarrollo progresivo y la codificación de esta área del derecho internacional. ${ }^{3}$ El programa de trabajo original, iniciado en 1955, se orientó hacia las consecuencias derivadas del incumplimiento de obligaciones internacionales. El análisis se extendió hasta 2001, cuando la Comisión aprobó el "Proyecto de Artículos sobre Responsabilidad del Estado por hechos internacionalmente ilícitos" y la Asamblea General tomó nota del texto y lo señaló a la atención de los miembros. ${ }^{4}$

En paralelo con esta labor, en 1970 la CDI decidió iniciar un nuevo camino de trabajo:

La Comisión procedería en primer lugar a examinar la cuestión de la responsabilidad de los Estados por hechos ilícitos internacionales. Se proponía estudiar por separado la cuestión de la responsabilidad derivada de determinados actos lícitos, tales como las actividades espaciales y nucleares, tan pronto como se lo permitiera su programa de trabajo. El fundamento totalmente distinto de la llamada responsabilidad por riesgos, la naturaleza diferente de las normas que la determinaban, así como de su contenido y de las formas que podía adoptar, harían que un examen simultáneo de ambas materias hiciera más difícil la comprensión de cada una de ellas. ${ }^{5}$

2 Barboza, Julio, "La responsabilidad sine delicto en el derecho internacional", Revista Jurídica de Buenos Aires, 2012, p. 52.

3 Crawford lo sintetizó con esta frase: "The ILC in codifying the law of state responsibility had to lay down general rules, which to some extent involved inventing them. The rules of state responsibility have been derived from cases, from practice, and from often unarticulated instantiations of general legal ideas". Cfr. Crawford, James, "The International Court of Justice and the Law of State Responsibility”, en Tams, Christian J. y Sloan, James (eds.), The Development of International Law by the International Court of Justice, Oxford University Press, 2013, p. 74.

4 A/RES/56/83 (12/12/2001). En adelante, el "Proyecto sobre Responsabilidad de 2001".

5 Comisión de Derecho Internacional, Anuario de la CDI 1970, Nueva York, Naciones Unidas, 1972, vol. II, p. 331, párr. 66 b). 
En apoyo de este nuevo enfoque, en 1973 la Asamblea General recomendó a la Comisión que emprendiera un estudio separado referente al tópico de la responsabilidad internacional por las consecuencias perjudiciales derivadas del ejercicio de otras actividades, es decir, sin la violación de una obligación internacional. ${ }^{6}$ Rosenne recordó que al votarse por este proyecto de resolución en la Sexta Comisión el resultado fue casi un empate: 42 votos a favor, 40 en contra y 21 abstenciones. En su opinión, eso representó un anticipo político de las dificultades que presentaría posteriormente el estudio de la temática. ${ }^{7}$

Esta línea de trabajo, inicialmente identificada como "Responsabilidad internacional por las consecuencias perjudiciales de actos no prohibidos por el derecho internacional", fue posteriormente segmentada. ${ }^{8}$ La CDI consideró prioritario analizar las reglas en materia de prevención de daños transfronterizos causados por actividades peligrosas y, en una etapa posterior, considerar la responsabilidad internacional en caso de pérdida provocada por ese mismo tipo de daños. ${ }^{9}$ El primer tramo quedó finalizado en 2001 mediante el "Proyecto de Artículos sobre prevención del daño transfronterizo resultante de actividades peligrosas", ${ }^{10}$ si bien la Comisión siguió considerando este tópico hasta 2007. ${ }^{11}$ Por su parte, el segundo tramo fue concluido en 2006 e iden-

6 A/RES/3071(XXVIII) (30/11/1973). De acuerdo con el texto en inglés: "The General Assembly... Recommends that the International Law Commission should... c) Undertake at an appropriate time a separate study of the topic of international liability for injurious consequences arising out of the performance of other activities". Acerca del uso de la expresión international liability y su traducción en otros idiomas, véanse Boyle, Alan E., "State Responsibility and International Liability for Injurious Consequences of Acts Not Prohibited by International Law: A Necessary Distinction?", The International and Comparative Law Quarterly, Cambridge, vol. 39, núm. 1, enero de 1990, pp. 8-10, y Barboza, Julio, The Environment, Risk and Liability in International Law, Leiden, Martinus Nijhoff, 2011, pp. 21-29.

7 Rosenne, Shabtai, The Perplexities of Modern International Law, Leiden, Martinus Nijhoff, 2004, p. 396.

8 Comisión de Derecho Internacional, Anuario de la CDI 1997, Nueva York-Ginebra, Naciones Unidas, 2002, vol. I, pp. 158-164, y A/RES/52/156 (26/01/1998).

9 Véanse las explicaciones del relator especial Rao, Pemmaraju Sreenivasa, "International Liability for Transboundary Harm”, Environmental Policy and Law, Ámsterdam, vol. 34, 2004, pp. 225 y 226.

10 Official Records of the General Assembly, Fifty-Sixth Session, Supplement No. 10 (A/ 56/10). La Asamblea General agradeció el trabajo realizado y encomendó a la CDI que siguiera ocupándose de esta temática, mediante la A/RES/56/82 (12/12/2001).

11 El texto del Proyecto de Artículos fue incorporado como anexo en A/RES/62/68 (06/ 12/2007). En adelante, el "Proyecto sobre Prevención de 2001-2007". 
tificado como "Proyecto de Principios sobre la asignación de la pérdida en caso de daño transfronterizo resultante de actividades peligrosas". ${ }^{12}$

Para una adecuada contextualización del trabajo de la CDI, se debe señalar que uno de los ejes principales sobre el cual se articulan los tres proyectos mencionados es la distinción entre las normas primarias y las secundarias. En el comentario a su Proyecto sobre Responsabilidad de 2001, la Comisión explicó:

El análisis insiste sobre todo en las normas secundarias de la responsabilidad del Estado, es decir, en las condiciones generales que han de satisfacerse, en derecho internacional, para que el Estado sea considerado responsable de acciones u omisiones ilícitas, y en las consecuencias jurídicas que nacen de esas acciones u omisiones. No se intenta definir en ellos el contenido de la violación de obligaciones internacionales que da lugar a la responsabilidad. Esta es la función de las normas primarias, cuya codificación entrañaría la reformulación de la mayor parte del derecho internacional sustantivo, tanto consuetudinario como convencional. ${ }^{13}$

En otras palabras, el derecho internacional determina que a los Estados les son exigibles ciertas conductas (ya sea de dar, hacer o no hacer), que son identificadas como obligaciones primarias. Si estas reglas son violadas, cobran vigencia las obligaciones secundarias, que se enfocan en las condiciones y consecuencias que se derivan del incumplimiento de las obligaciones preexistentes. ${ }^{14}$ Sin embargo, este enfoque varía al considerar la responsabilidad sine delicto. Barboza explicó que, de producirse un daño originado en una actividad lícita, existe una obligación primaria que involucra el pago de

12 A/RES/ $61 / 36$ (04/12/2006). En adelante, el "Proyecto de Principios de 2006".

13 Comisión de Derecho Internacional, Anuario de la CDI 2001, vol. II: Segunda parte, Nueva York-Ginebra, Naciones Unidas, 2007, p. 31.

14 Nuevamente, en palabras de la Comisión: “...una cosa era definir una norma y el contenido de la obligación por ella impuesta y otra muy distinta determinar si se había infringido esa obligación y cuáles debían ser las consecuencias de tal infracción. Únicamente este segundo aspecto entraba en el ámbito de la responsabilidad; cualquier confusión al respecto sería un obstáculo que podría frustrar una vez más las esperanzas de codificar la materia”. Comisión de Derecho Internacional, Anuario de la CDI 1970, cit., p. 331, párr. 66 c). De acuerdo con David: "Fundamentally, this distinction... was adopted to serve a precise practical objective: to allow the Commission to focus solely on the structural rules of international responsibility, to the exclusion of the substantive rules the violation of which gives rise to responsibility". Cfr. David, Eric, "Primary and Secondary Rules", en Crawford, James et al. (eds.), The Law of International Responsibility, Oxford-Nueva York, Oxford University Press, 2010, p. 28. 
una compensación por ese perjuicio. Si esta regla fuera violada, surgiría una obligación secundaría, que constituye la consecuencia de la afectación de la primera. ${ }^{15}$ La propia CDI sostuvo esta perspectiva, al presentar el Proyecto de Principios de 2006:

El presente proyecto de principios, como el proyecto de artículos sobre la prevención, versan sobre normas primarias. Por consiguiente, el incumplimiento de la obligación de prevención que establece el proyecto de artículos sobre la prevención podría dar lugar a la responsabilidad del Estado por hecho ilícito (State responsibility) sin que ello implique necesariamente que la actividad misma esté prohibida. En tal caso, se podría invocar la responsabilidad del Estado (State responsibility) para hacer efectivas, no sólo las obligaciones del Estado mismo, sino también la responsabilidad civil u obligación del explotador. ${ }^{16}$

Una vez expuesto el trabajo desarrollado por la CDI, se debe advertir que, junto con las razonables dificultades teóricas que afrontó al elaborar sus tres proyectos, también encontró una gran resistencia por parte de muchos miembros de las Naciones Unidas, quienes serían los destinatarios últimos de estas reglas.

Respecto del Proyecto sobre Responsabilidad de 2001, se observa la divergencia entre las opiniones gubernamentales, tanto respecto de la redacción de varios artículos como acerca de la conveniencia de adoptar un tratado en la materia y la forma en que podría concretarse. Los Estados que aportaron su opinión, en reducida cantidad, presentan un amplio espectro, desde el rechazo a crear un instrumento vinculante, incluyendo críticas a la redacción del Proyecto (Alemania, Australia, Estados Unidos, Países Bajos, Qatar y Reino Unido), a la necesidad de adoptar un tratado a través de una

15 Barboza, Julio, "Sine Delicto (Causal) Liability and Responsibility for Wrongful Acts in International Law”, en International Law Commission, International Law on the Eve of the TwentyFirst Century. Views from the International Law Commission, Nueva York, Naciones Unidas, 1997, p. 324. Véase también la opinión de Gómez-Robledo Verduzco, Alonso, Responsabilidad internacional por daños transfronterizos, 2a. ed., México, UNAM, 2014, pp. 46-48.

16 Comisión de Derecho Internacional, Anuario de la CDI 2006, vol. II: Segunda parte, Nueva York-Ginebra, Naciones Unidas, 2013, p. 69, párr. 6). A causa de esta interacción entre ambas esferas de la responsabilidad, es que Sucharitkul las describió como intrínsecamente interconectadas. Cfr. Sucharitkul, Sompong, "State Responsibility and International Liability under International Law", Loyola of Los Angeles International and Comparative Law Journal, Los Ángeles, vol. 18,1996 , p. 838 . 
Esta revista forma parte del acervo de la Biblioteca Jurídica Virtual del Instituto de Investigaciones Jurídicas de la UNAM

conferencia internacional o por otros medios (Austria, Brasil, Chile, El Salvador, Francia, Lituania, México y Portugal), o mantener el texto del Proyecto como anexo de una resolución de la Asamblea General, rechazando la convocatoria de una conferencia internacional (República Checa, Dinamarca, Finlandia, Islandia, Noruega y Suecia). ${ }^{17}$

El quinto y último relator especial de la CDI en este campo, James Crawford, sopesó las opiniones favorables y las críticas hacia el Proyecto sobre Responsabilidad de 2001, señalando que, en términos generales, los apoyos habían sido significativos. ${ }^{18}$ Asimismo, Crawford destacó que la consolidación de este texto llegaría a través de su aplicación continua por los tribunales internacionales. ${ }^{19}$ Esta expresión de deseos aparece inicialmente cumplida, ya que la jurisprudencia internacional reconoció el carácter consuetudinario de varios artículos del Proyecto. ${ }^{20}$

Ahora bien, con relación a la responsabilidad sine delicto, su marco jurídico no presenta el mismo nivel de aceptación, situación reconocida por Barboza cuando expresó que no tenía carta de ciudadanía en el derecho internacional general. ${ }^{21}$ Es sólo respecto de materias específicas que los Estados acordaron

17 Informes del secretario general de la ONU, de título "Responsabilidad del Estado por hechos internacionalmente ilícitos. Información y observaciones recibidas de los gobiernos”, A/62/63 (9/03/2007), A/65/96 (14/05/2010), A/68/69 (27/03/2013), A/68/69/Add.1 (28/06/2013) y A/71/79 (21/04/2016). Como simple referencia, los comentarios del gobierno austríaco (A/71/79, p. 3) son elocuentes de la situación actual: "Austria, en principio, estaría a favor de la aprobación de una convención. Sin embargo, el proyecto de una convención se debería intentar conseguir solo si hay suficientes garantías de que la estructura actual y el equilibrio de los proyectos de artículo se mantendrán y de que se evitará un debate renovado de sus disposiciones sustanciales; y si existen perspectivas realistas de que una convención de ese tipo logrará una amplia ratificación y aceptación”.

18 Un interesante trabajo que expone algunas de las críticas al Proyecto se puede ver en Yamada, Chusei, "Revisiting the International Law Commission's Draft Articles on State Responsibility”, en Ragazzi, Maurizio (ed.), International Responsibility Today. Essays in Memory of Oscar Schachter, Leiden, Koninklijke Brill NV, 2005, pp. 117-123. El autor se desempeñó como miembro de la CDI durante la discusión en primera y segunda lectura del Proyecto, de ahí su gran conocimiento de la temática desarrollada.

19 Crawford, James, State Responsibility: The General Part, Cambridge University Press, 2013, pp. 42-44 y 85-92.

20 Son ilustrativos los informes producidos por el secretario general de la ONU, de título "Responsabilidad del Estado por hechos internacionalmente ilícitos. Compilación de las decisiones de cortes, tribunales y otros órganos internacionales”, A/68/72 (30/04/2013) y A/71/80 (21/04/2016).

21 Barboza, Julio, “La responsabilidad...”, op. cit., p. 52. 
sistemas de resarcimiento, basados en el daño y sin que presupongan la violación del derecho vigente. ${ }^{22}$ En concordancia, la recepción de los Estados al Proyecto sobre Prevención de 2001-2007 y al Proyecto de Principios de 2006 fue considerablemente más cautelosa que respecto del texto sobre responsabilidad por hechos ilícitos.

Los escasos comentarios y observaciones remitidos a la CDI por los miembros de la ONU pueden reflejar tanto una falta de consenso como un desinterés puntual en una legislación general para este campo. ${ }^{23}$ Con relación al Proyecto sobre Prevención de 2001-2007, sólo cinco Estados aportaron sus puntos de vista (Francia, Líbano, Países Bajos, Turquía y Reino Unido), y no hicieron referencia a la posible aprobación como norma vinculante. De hecho, varios de ellos, si bien felicitaron a la CDI por su labor, también criticaron los postulados del Proyecto. ${ }^{24}$ Respecto del Proyecto de Principios de 2006, nueve Estados remitieron comentarios a la CDI (República Checa, Líbano, México, Países Bajos, Pakistán, Siria, Reino Unido, Estados Unidos y Uzbekistán) y se expresaron en forma dubitativa con relación a los principios propuestos, e incluso remarcaron que se trataba de una normativa con un neto carácter de desarrollo progresivo. ${ }^{25}$ Por su parte, al ser debatidos estos tópicos en la Sexta Comisión de la Asamblea General, las opiniones volvie-

22 Véase el informe elaborado por el secretario general, de título "Estudio de los regímenes de responsabilidad relacionados con el tema de la responsabilidad internacional por las consecuencias perjudiciales de actos no prohibidos por el derecho internacional", A/CN.4/543 (24/06/2004). En doctrina, véanse la compilación parcial realizada por Descamps, Hannes et al. International Documents on Environmental Liability, Heidelberg, Springer Netherlands, 2008, y el análisis de los modelos de responsabilidad que se aplican en cada instrumento en Rosas, Allan, "Issues of State Liability for Transboundary Environmental Damage", Nordic Journal of International Law, Leiden, vol. 60, núm. 2, 1991, pp. 29-47.

23 Como sintetizó Foster: "The common view amongst States was that either an activity was prohibited, in which case the law on State responsibility would govern any reparation to be made in respect of its consequences; or an activity was not prohibited and no international legal responsibility or liability could attach to the effects produced by the activity". Cfr. Foster, Caroline, "The ILC Draft Principles on the Allocation of Loss in the Case of Transboundary Harm Arising out of Hazardous Activities: Privatizing Risk?”, Review of European Community \& International Environmental Law, Nueva Jersey, vol. 14, núm. 3, 2005, p. 265.

24 Líbano lo consideró contradictorio e incompleto, y los Países Bajos manifestaron que se encontraban decepcionados por su texto. Véanse A/CN.4/509 (sesión del 01/05 al 09/ 06/2000 y del 10/07 al 18/08/2000) y A/CN.4/516 (sesión del 23/04 al 01/06/2001 y del $02 / 07$ al 10/08/2001).

25 A/CN.4/562 y Add.1 (del 01/05 al 09/06/2006 y del 03/07 al 11/08/2006). 
Esta revista forma parte del acervo de la Biblioteca Jurídica Virtual del Instituto de Investigaciones Jurídicas de la UNAM

ron a estar divididas en cuanto a sus características intrínsecas y, en especial, respecto de la necesidad de adoptarlo como un instrumento obligatorio, posición esta última sostenida por el representante de Argentina. ${ }^{26}$

La recepción por parte de la doctrina también fue disímil, desde algunas posiciones eclécticas hasta otras francamente desfavorables. ${ }^{27}$ Birnie et al. afirmaron que el Proyecto sobre Prevención de 2001-2007 es esencialmente codificatorio:

The 2001 Articles on Transboundary Harm essentially codify existing obligations of environmental impact assessment, notification, consultation, monitoring, prevention, and diligent control of activities likely to cause transboundary harm. These articles are securely based in existing precedents... [they] offer an authoritative exposition of the existing law. Not surprisingly, they have been heavily cited by parties to international environmental litigation. ${ }^{28}$

26 A/C.6/62/SR.12 (23/10/2007) y A/C.6/62/SR.28 (19/11/2007). La intervención del representante argentino se encuentra en la página 5 del primer documento citado. El debate continuó en las sucesivas sesiones de la Sexta Comisión: A/C.6/65/SR.17 (21/10/2010), A/C.6/65/SR.27 (5/11/2010), A/C.6/71/SR.18 (20/10/2016), A/C.6/71/SR.31 (4/11/ 2016) y A/C.6/71/SR.32 (7/11/2016). Como diplomáticamente señaló el representante de la India (A/C.6/65/SR.17, p. 6): "Los proyectos de artículos y de principios sirven, cada uno de ellos, como guía útil a los Estados en la adopción de medidas legislativas, reglamentarias y administrativas para incorporar los principios pertinentes en su legislación y sus políticas internas. Todo intento de codificar el proyecto de artículos en forma de una convención sería contraproducente y no agregaría nada al desarrollo progresivo del derecho internacional ya logrado sobre este tema. La práctica de los Estados indica una preferencia por los tratados ambientales sectoriales con regímenes de responsabilidad”.

27 Además de los textos que serán mencionados en los párrafos subsiguientes, es posible citar, de manera totalmente enunciativa, los trabajos de Handl, Günther, "Territorial Sovereignty and the Problem of Transnational Pollution", The American Journal of International Law, vol. 69, núm. 1, enero de 1975, pp. 50-76; Handl, Günther, "State Liability for Accidental Transnational Environmental Damage by Private Persons", The American Journal of International Law, vol. 74, núm. 3, julio de 1980, pp. 525-565; Magraw, Daniel Barstow, "Transboundary Harm: The International Law Commission's Study of «International Liability»”, The American Journal of International Law, vol. 80, núm. 2, abril de 1986, pp. 305-330; Dupuy, Pierre-Marie, “The International Law of States Responsibility: Revolution or Evolution?", Michigan Journal of International Law, Míchigan, vol. 11, 1989, pp. 105-128.

28 Birnie, Patricia et al., International Law and the Environment, 3a. ed., Oxford University Press, 2009, p. 141. Los autores referencian a los litigios en The MOX Plant (Ireland v. United Kingdom), Permanent Court of Arbitration case 2002-01, y Pulp Mills on the River Uruguay (Argentina v. Uruguay), I.C.J. Reports 2006, p. 113. 
Por el contrario, para estos autores, el Proyecto de Principios de 2006 implicaría un ejercicio de desarrollo progresivo: "While the 2006 ILC Principles as a whole cannot be viewed as an exercise in codifying customary international law, they show how the Commission has made use of general principles of law as «an indication of policy and principle»". ${ }^{29}$

En forma totalmente opuesta, Remiro Brotóns expresó elocuentemente su rechazo al enfoque de la responsabilidad sine delicto, opinión que nos permitimos transcribir in extenso:

Basándose, precisamente, en la interpretación de algunas decisiones arbitrales (Fundición de Trail, 1941, Lago Lanós, 1957) y judiciales (CIJ, Estrecho de Corfú, 1949), se ha sostenido doctrinalmente que la obligación de reparar los daños a terceros resultantes de actividades no prohibidas forma ya parte del DI general. Se ha hablado así de una responsabilidad por riesgo, considerando que surge de la realización de actividades especialmente peligrosas, o de una responsabilidad absoluta, en tanto que no admite excepciones. Sin embargo, lo que se deduce de las decisiones invocadas es, más bien, y así lo entendía el antiguo Relator de la CDI en la materia, J. Barboza, que los Estados tienen la obligación de no permitir que sus territorios sean utilizados para la realización de actos contrarios a los derechos de otros Estados, lo que constituye una obligación general de prevención, cuya violación generaría responsabilidad. ${ }^{30}$

Otros autores no rechazan en forma categórica el concepto de una responsabilidad sine delicto como Remiro Brotóns, pero sí acotan su contenido a los tratados específicos, sin admitirla como un principio general del derecho internacional. Entre ellos, Diez de Velasco, citando a Marín López, señaló que “... las reglas y principios [de derecho internacional] relativos a este nuevo tipo de responsabilidad surgida a raíz de los avances tecnológicos distan

29 Ibidem, p. 321. Similar opinión expresó Handl, Günther, "International Accountability for Transboundary Environmental Harm Revisited: What Role for «State Liability»?”, Environmental Policy and Law, Ámsterdam, vol. 37, 2007, pp. 121 y 122.

30 El jurista español agrega: "Estamos, pues, también ahora ante una responsabilidad generada por la infracción de una obligación primaria, establecida en su caso convencionalmente, la de realizar actividades ultra-peligrosas acogiéndose estrictamente al cumplimiento de los estándares de diligencia debida. Por ello, a salvo regímenes convencionales, no hay base consuetudinaria suficiente que consagre una responsabilidad sin ilícito”. Remiro Brotóns, Antonio, Derecho internacional, Valencia, Tirant Lo Blanch, 2007, pp. 747 y 748. 
de estar bien establecidos y requieren más precisos desarrollos". ${ }^{31}$ En igual sentido, Rezek expresó que no se admite en el derecho de gentes una responsabilidad objetiva, excepto en casos especiales y materias reguladas por convenciones modernas. ${ }^{32}$ Por su parte, Xue criticó la intención de traspasar en forma directa las reglas que surgen de tratados específicos, para convertirlas en una regla general: "To harmonize such arrangements on a global basis requires more consistent State practice and further development of international law on international liability in each and every field concerned". ${ }^{33}$

A modo de cierre para este apartado, creemos que es interesante retomar el comentario de Barboza que encabezó estas líneas. Las reglas que regulan la responsabilidad internacional por hechos ilícitos aparecen consolidadas gracias al Proyecto sobre Responsabilidad de 2001. Si bien este texto no está exento de algunas críticas, hasta el momento los importantes apoyos explícitos o implícitos demuestran su aceptación como el reflejo parcial del derecho consuetudinario. Por el contrario, el concepto de responsabilidad sine delicto, trabajosamente desarrollado por la CDI en sus proyectos de 20012007 y 2006, no cuenta con una recepción tan favorable. Por el momento, se trataría de reglas generales en desarrollo que, partiendo del antecedente de convenciones específicas, buscan consolidarse a través de la práctica estatal.

\section{DESARROLLO DE LA CONTROVERSIA}

POR EL VERTEDERO DE RESIDUOS

La Cordillera de los Andes constituye la frontera natural entre Argentina y Chile, extendiéndose de norte a sur por más de 5,000 kilómetros. Desde su independencia a comienzos del siglo XIX, ambos Estados suscribieron diversos tratados para fijar el límite internacional entre las respectivas soberanías. A pesar de este esfuerzo, los conflictos por este espacio geográfico

31 Diez de Velasco, Manuel, Instituciones de derecho internacional público, Madrid, Tecnos, 2007, p. 864, con cita a Marín López, Antonio, “Aspectos actuales de la responsabilidad internacional”, en Miaja de la Muela, Adolfo et al. (comp.), Estudios de derecho internacional. Homenaje al Prof. Miaja de la Muela, Madrid, Tecnos, 1979, t. II, pp. 815-836.

32 Rezek, Francisco, Direito internacional público, 11a. ed., São Paulo, Saraiva, 2008, p. 270.

33 Xue, Hanqin, Transboundary Damage in International Law, Cambridge University Press, 2003, pp. 320 y 321. 
fueron innumerables, y aún en la actualidad se producen ocasionalmente algunas divergencias.

El conflicto bajo análisis tuvo su inicio formal en mayo de 2014, cuando la empresa minera argentina Xstrata Pachón S. A. presentó una demanda civil en la justicia federal de la Provincia de San Juan contra la empresa chilena Minera Los Pelambres, por la ocupación indebida de su propiedad y el daño ambiental ahí provocado. ${ }^{34}$ En su exposición de los hechos, la denunciante explicó que ambas empresas poseían áreas de explotación en espacios linderos de la Cordillera de los Andes, separadas por el límite internacional entre Argentina y Chile. En este contexto, Xstrata Pachón S. A. denunció que, al realizar una inspección en la zona del Cerro Amarillo en diciembre de 2013, se descubrió un vertedero de residuos, ${ }^{35}$ que la empresa chilena había instalado ilegalmente. Es decir, una porción de los materiales sin utilidad que Minera Los Pelambres extraía de la Cordillera eran depositados en territorio argentino, en el área de explotación concedida a la empresa denunciante. Asimismo, Xstrata Pachón S. A. advirtió que el material en la escombrera había iniciado un proceso de degradación, producto de diversos factores climáticos y de la falta de tratamiento adecuado, generándose un drenaje ácido de roca. Adicionalmente, a causa de la construcción de ductos dentro de la escombrera, se había producido un desvío de aguas que naturalmente deberían escurrirse en territorio argentino, con el consecuente deterioro de las lagunas cercanas a las áreas de explotación. En síntesis, además de la ocupación ilegal del predio de la empresa denunciante (y la violación de la soberanía territorial argentina), la actividad de la empresa chilena provocaba un grave daño ambiental transfronterizo.

En su presentación en el expediente, Minera Los Pelambres reconoció la existencia del vertedero, que había sido utilizado entre 2007 y 2011, pero justificó su conducta a partir de una confusión respecto de los límites internacionales. Esta empresa chilena explicó que la concesión sobre su área de explotación se había realizado teniendo en cuenta la cartografía chilena, que ubicaba al sector de la escombrera en dicho país. La empresa demandada afirmó que, en 2012, con posterioridad al vertido de residuos en Cerro Amarillo, Argentina y Chile habían acordado una modificación en el límite

34 Causa FMZ 13.033/2014, "Xstrata Pachón S. A. c/ Minera Los Pelambres s/ ordinario”, Juzgado Federal de San Juan $\mathrm{N}^{\circ} 1$.

35 También es identificado como botadero o escombrera, expresiones que, a los fines de este trabajo, serán usadas en forma indistinta. 
internacional, lo que derivó en que el depósito de material en desuso quedara ubicado en territorio argentino.

El 20 de marzo de 2015, Xstrata Pachón S. A. presentó una nueva denuncia, esta vez en el fuero penal, por el delito de usurpación y por el accionar ilícito configurado por el vertido de residuos tóxicos en su propiedad. ${ }^{36}$

Las autoridades judiciales actuantes solicitaron diversos informes al Poder Ejecutivo argentino, de los cuales son de gran relevancia los requeridos a la Comisión Nacional de Límites, dependiente del Ministerio de Relaciones Exteriores, Comercio Internacional y Culto, y al Ministerio de Medio Ambiente.

En la respuesta aportada por la Comisión Nacional de Límites se rechazó la versión ofrecida por Minera Los Pelambres, explicando que, en el sector en conflicto, el límite internacional había sido establecido por el Tratado del 23 de julio de 1881, y que la demarcación única y definitiva se había realizado en 1901, erigiendo una serie de hitos. La Comisión aclaró que el trabajo realizado a nivel bilateral en 2012 era simplemente una mejora en la demarcación en la zona limítrofe, actividad que se conoce como densificación de la frontera. ${ }^{37}$

Por su parte, al Ministerio de Medio Ambiente se le pidió que determinara la veracidad de los hechos denunciados por Xstrata Pachón S. A. y que constatara la existencia de contaminación en el territorio argentino, originada por las actividades de la empresa chilena. El Ministerio produjo una pericial el 6 de julio de 2017, en la cual expresó: "En términos de dimensionamiento, la Escombrera Cerro Amarillo se extiende dentro del territorio argentino ocupando una superficie de 52 hectáreas, y un volumen aproximado de 20 millones de metros cúbicos". ${ }^{38}$ Además, el Ministerio señaló: "Se observó una afectación directa de la escombrera sobre las lagunas del lado argentino por disminución de superficie del 53\%...” y que “...es posible concluir que la presencia de la Escombrera Cerro Amarillo constituye en la actualidad una fuente cierta de dispersión de sustancias contaminantes...". ${ }^{39}$

36 Causa FMZ 6003/2015, "C.N.N. - sobre infracción Ley 24.051, art. 181 inc. 1) y art. 182 inc. 1) del Código Penal”, Juzgado Federal de San Juan $\mathrm{N}^{\circ} 2$.

$37 \mathrm{Al}$ respecto, véase Barberis, Julio, El territorio del Estado y la soberanía territorial, Buenos Aires, Ábaco, 2003, pp. 175-177.

38 República Argentina, Ministerio de Ambiente y Desarrollo Sustentable, "Informe pericial del 06/07/2017. Escombrera Cerro Amarillo de Minera Pelambres”, p. 13.

39 Ibidem, p. 15. 
Sobre la base de la información recibida, las autoridades judiciales dieron por acreditada la usurpación del espacio concedido a Xstrata Pachón S. A., y la afectación de la soberanía territorial argentina, y el daño ambiental transfronterizo. En consecuencia, el Juzgado Federal de San Juan $\mathrm{N}^{\circ} 2$ dictó una orden el 3 de agosto de 2017 con una serie de medidas destinadas a la demandada, incluyendo la compactación de la superficie de la escombrera para permitir el escurrimiento de las aguas y el establecimiento de un sistema de drenaje, de modo que las aguas escurridas no entraran en contacto con las aguas superficiales de la zona. Asimismo, el Juzgado ordenó que se retirara la totalidad de la escombrera, con la mayor celeridad posible. ${ }^{40}$

\section{SOLUCIÓN DEL DIFERENDO \\ ENTRE ARGENTINA Y CHILE}

Como fuera mencionado, las cuestiones limítrofes siempre fueron uno de los aspectos álgidos en la relación bilateral entre ambos países vecinos. Por este motivo, los gobiernos acordaron crear una Comisión de Alto Nivel para encauzar la problemática de la escombrera y del daño transfronterizo, elevando al plano político y diplomático la hasta entonces disputa privada entre dos empresas.

La Comisión mantuvo cuatro reuniones durante 2017: dos en Buenos Aires (24 de mayo y 11 de septiembre) y dos en Santiago de Chile (8 de agosto y 11 de octubre). Como resultado de las negociaciones desarrolladas en esos encuentros, se convino en solucionar la disputa mediante la firma de dos tratados internacionales.

El primer acuerdo tuvo origen en una nota enviada a las autoridades argentinas por la Embajada de la República de Chile en Buenos Aires, el 6 de diciembre de 2017. En ella se propuso habilitar un paso fronterizo provisorio, para que empleados de Minera Los Pelambres ingresaran al territorio argentino a retirar el material descartado sin autorización. Esta acción per-

40 Un racconto del caso, desde la visión de las autoridades del Ministerio Público de la Provincia de San Juan, en Noguera Ramos, Gastón, "Caso «Pelambres»: usurpación, conflictos de límites y potencialidad de daño”, Revista de Derecho Ambiental, Buenos Aires, núm. 53, 2018 , pp. $121-131$. 
mitiría dar cumplimiento a la orden de la autoridad judicial argentina, en el sentido de iniciar los trabajos necesarios para retirar la escombrera con la mayor celeridad posible. Los términos de esta propuesta fueron aceptados por el ministro de Relaciones Exteriores de Argentina mediante una nota del 7 de diciembre de 2017.

El segundo acuerdo se concretó nuevamente a través de cartas reversales, en un único día, el 12 de diciembre de 2017, por medio de una nota de propuesta, remitida por la Embajada de Chile en Buenos Aires, y aceptada íntegramente por la República Argentina en su nota de respuesta. En el texto acordado se menciona que las autoridades chilenas habían pactado con Minera Los Pelambres la realización de un plan de obras, que incluía el retiro de la escombrera del territorio argentino y la concreción de trabajos de mitigación ambiental. Este plan de trabajo ya había sido presentado ante las autoridades judiciales argentinas, quienes habían manifestado su aceptación. En apoyo de estas acciones, Argentina y Chile, como partes en el acuerdo, dispondrían las medidas necesarias para contribuir a la ejecución urgente del plan, de conformidad con su derecho interno. Además, las partes realizarían un seguimiento de la situación y se transmitirían toda la información pertinente a través de sus respectivos ministerios de relaciones exteriores. De esta manera, “...para la República Argentina el cumplimiento del retiro completo del botadero «Cerro Amarillo» y la realización satisfactoria de las obras de mitigación ambiental asociadas constituyen una solución definitiva, y que resulta ambientalmente adecuada”.

Asimismo, el penúltimo párrafo de este segundo acuerdo estipula:

Nada de lo expresado en esta Nota, así como en la Nota de respuesta, altera la posición jurídica que mantiene cada Estado, conocida por ambos y que reservan expresamente, respecto de las implicancias jurídicas derivadas para los Estados de la presencia del botadero "Cerro Amarillo" en territorio argentino.

La estipulación transcripta implicaría una reserva de futuros reclamos por parte de Argentina, vinculados tanto con la violación de su soberanía como con el problema de la contaminación transfronteriza sufrida. Sin embargo, hasta la fecha de redacción de estas líneas, no existen indicios acerca de una posible reapertura del conflicto. 


\section{LA CONTROVERSIA DE LA CORDILLERA A LA LUZ}

DE LA RESPONSABILIDAD INTERNACIONAL

$\mathrm{Al}$ analizar el caso Corfu Channel ${ }^{41}$ como un paradigma en materia de responsabilidad, Brownlie señaló: "This decision provides a very helpful demonstration of the necessary transition from the generalities of the literature to the particular problems of the marshaling of evidence, the standard of proof and the application of the relevant legal principles in particular situations". ${ }^{42}$

En otras palabras, si un conflicto actual entre Estados es analizado exclusivamente desde un enfoque teórico, pueden perderse de vista las diferentes cuestiones prácticas involucradas, entre ellas, como expresó el autor inglés, los métodos de obtención de evidencia para sostener una posición frente a los jueces y el estándar de prueba exigido por el tribunal.

Esta lógica parece primar en las controversias que involucran un daño ambiental transfronterizo. Desde la teoría parecería simple llevar adelante un litigio internacional de estas características, identificando al Estado en el cual se origina el daño, denunciando al operador o entidad contaminante, y aportando evidencia del perjuicio sufrido. Sin embargo, en la práctica existen diferentes complicaciones que, al ser ponderadas por el Estado afectado, lo obligan a repensar la forma de alegar las reglas de responsabilidad internacional para dirimir la cuestión.

La reducida casuística jurisdiccional internacional en los asuntos ambientales confirma la anterior observación. De acuerdo con Loibl:

Although already in the 1940's the Trail Smelter Arbitration addressed transboundary environmental damages, the number of cases that have been brought to international adjudication has been limited. This is underlined by the fact that since the ICJ established a special Chamber for Environmental Matters in 1993, no case was referred to this Chamber. Furthermore, environmental issues have hardly been at

41 Corfu Channel Case, Judgment of April 4th, 1949, I.C.J. Reports 1949, p. 4.

42 Brownlie, Ian, "State Responsibility and the International Court of Justice", en Fitzmaurice, Malgosia y Sarooshi, Dan (eds.), Issues of State Responsibility before International Judicial Institutions, Oxford, Hart Publishing, 2004, p. 14. 
Esta revista forma parte del acervo de la Biblioteca Jurídica Virtual del Instituto de Investigaciones Jurídicas de la UNAM

the heart of disputes before other international courts or arbitral tribunals between States. ${ }^{43}$

\section{En palabras de Scovazzi:}

It is a matter of fact that States are rather reluctant to challenge each other in the field of international responsibility for environmental harm. This attitude is so rooted that it sometimes happens that States affected by environmental harm refrain from making claims of international responsibility because of the doubts about the success of such a step and, perhaps, because of the concern to establish a precedent that could play against them in future cases. ${ }^{44}$

Esas dudas que plantean los Estados acerca del éxito de un litigio por daño transfronterizo, como mencionó Scovazzi, encontrarían su justificación al menos en dos cuestiones: primero, porque las reglas en materia de responsabilidad sine delicto no cuentan con una aceptación extendida, y no se les considera, por el momento, como parte del derecho internacional general, y, segundo, a causa de las dificultades que pueden surgir para reunir la prueba suficiente, que permita endilgar la responsabilidad a un Estado, en muchos

43 Loibl, Gerhard, "Environmental Law and Non-Compliance Procedures: Issues of State Responsibility”, en Fitzmaurice, Malgosia y Sarooshi, Dan (eds.), Issues of State Responsibility before International Judicial Institutions, Oxford, Hart Publishing, 2004, p. 202. En contra, véase Sands, Philippe, "Litigating Environmental Disputes: Courts, Tribunals and the Progressive Development of International Environmental Law”, Environmental Policy and Law, Ámsterdam, vol. 37, 2007, pp. 67 y 68, quien destacó el incremento de jurisdicciones internacionales avocadas a la temática ambiental, así como el interés de diferentes actores en iniciar litigios en este campo. Si bien es acertado que, con posterioridad a la publicación de Loibl en 2004, las jurisdicciones internacionales sumaron algunos aportes a la temática ambiental, los precedentes continúan siendo escasos. En la nómina de la Corte Internacional de Justicia se puede contabilizar al ya mencionado Pulp Mills on the River Uruguay (Argentina v. Uruguay); Whaling in the Antarctic (Australia v. Japan: New Zealand Intervening), Judgment, I.C.J. Reports 2014, p. 226; Certain Activities Carried Out by Nicaragua in the Border Area (Costa Rica v. Nicaragua) y Construction of a Road in Costa Rica along the San Juan River (Nicaragua v. Costa Rica), Judgment, I.C.J. Reports 2015, p. 665, y Dispute over the Status and Use of the Waters of the Silala (Chile v. Bolivia), este último aún en desarrollo. Un litigio sobre daño ambiental transfronterizo, presentado y luego removido de la lista de la Corte, es Aerial Herbicide Spraying (Ecuador v. Colombia), Order of 13 September 2013, I.C.J. Reports 2013, p. 278.

44 Scovazzi, Tullio, "Some Remarks on International Responsibility in the Field of Environmental Protection”, en Ragazzi, Maurizio (ed.), International Responsibility Today. Essays in Memory of Oscar Schachter, Leiden, Koninklijke Brill NV, 2005, p. 215. 
casos por la conducta perjudicial desplegada por una empresa del sector privado. ${ }^{45}$ En nuestra opinión, la disputa entre Argentina y Chile ejemplifica claramente ambas dificultades.

Una primera lectura de esta controversia parece situarnos rápidamente en el campo de la responsabilidad por las consecuencias perjudiciales de actividades lícitas; es decir, que en el desarrollo de una actividad industrial (minería a gran escala), autorizada por el Estado territorial, se habría causado un perjuicio de manera transfronteriza a otro Estado. Asimismo, desde una perspectiva jurisdiccional, existirían muchas probabilidades de éxito, dada la notoria evidencia física del daño y el reconocimiento explícito hecho por la empresa contaminante.

Sin embargo, una lectura más profunda permite entrever ciertos aspectos que no encuadran de forma sencilla en la formulación teórica de la responsabilidad sine delicto y que, adicionalmente, dificultan las cuestiones probatorias. La CDI expresó de forma adecuada esta complejidad:

Hay que señalar que, al trasladar el concepto de responsabilidad objetiva de un contexto interno, nacional (donde está bien establecido, aunque con todas las diferencias relativas a la forma en que se invoca y se aplica en distintas jurisdicciones) para convertirlo en una norma internacional, se deben definir cuidadosamente sus elementos, aunque sin perder de vista el objetivo básico, a saber, imputar a una persona, sin ninguna prueba de culpa, la responsabilidad por haber creado un riesgo al dedicarse a una actividad peligrosa. ${ }^{46}$

En primer lugar, cabe observar que el daño transfronterizo fue producto de una acción deliberada de la empresa, que depositó los residuos de su explotación en el área oriental del límite internacional, fuera del espacio concedido por el Estado territorial para su actividad. De esta manera, no puede catalogarse como una consecuencia no deseada de la actividad industrial, o de un perjuicio causado por un accidente, cualquiera que sea su origen (hecho de la naturaleza o actividad humana). Por el contrario, la empresa

45 Nuevamente, en palabras de Scovazzi: "It follows that, once it has been determined that a private entity has failed to exercise due diligence in carrying out a harmful activity, it still needs to be determined whether a State organ omitted to exercise due diligence in controlling that private entity. Again, States are rather reluctant to claim or to accept international responsibility for a conduct which is not taken directly by their organs”. Ibidem, p. 216.

46 Comisión de Derecho Internacional, Anuario de la CDI 2006, cit., p. 88, párr. 17. 
chilena realizó un acto contrario a las normas vigentes, de ahí que las consecuencias que se le imputan se deriven de esa acción voluntaria. ${ }^{47}$

En segundo lugar, también es posible encuadrar el caso desde la violación por parte de Chile de su deber de prevención y supervisión, y derivar de esa conducta el perjuicio para Argentina. Por lo tanto, se trataría de un planteo clásico de responsabilidad internacional, como expresaba Remiro Brotóns ${ }^{48}$ como consecuencia de la violación de una obligación primaria, que establece que un Estado no puede permitir que su territorio sea utilizado para causar perjuicio a un tercer Estado. ${ }^{49}$ Es en este sentido que se justificaría la inclusión del penúltimo párrafo del Acuerdo del 12 de diciembre de 2017, cuando señala que la solución consensuada entre las partes no altera las posiciones respectivas con relación a las implicancias jurídicas de la presencia del botadero en territorio argentino. De acuerdo con una interpretación razonable de esa estipulación, y si bien el plan de obras permitiría remediar las consecuencias inmediatas e impedir un agravamiento de la situación, sería posible iniciar en fecha posterior una reclamación de Estado a Estado por la violación del derecho internacional. En opinión de la CDI:

...los Estados son responsables en derecho internacional del incumplimiento de sus obligaciones de prevención. Por ello, el proyecto de principios [de 2006] se entiende sin perjuicio de las normas relativas a la responsabilidad del Estado por hecho ilícito (State responsibility) y de cualquier pretensión aducida al amparo de esas normas en caso de violación de las obligaciones de prevención..$^{50}$

A su vez, debe quedar en claro que el Estado territorial no puede eximirse de responsabilidad interponiendo la figura de un particular. ${ }^{51} \mathrm{Si}$ bien la

47 En palabras de Xue, se podría aplicar la categoría de "daño transfronterizo no accidental”, que involucra aquellos casos en los cuales el daño es provocado por actos deliberados, ocasionales o acumulativos, con efectos perjudiciales. Xue, Hanqin, op. cit., p. 113.

48 Remiro Brotóns, Antonio, op. cit., p. 748.

49 En palabras de la Corte Internacional de Justicia: "The existence of the general obligation of States to ensure that activities within their jurisdiction and control respect the environment of other States or of areas beyond national control is now part of the corpus of international law relating to the environment". Cfr. Legality of the Threat or Use of Nuclear Weapons, Advisory Opinion, I.C.J. Reports 1996, p. 226.

50 Comisión de Derecho Internacional, Anuario de la CDI 2006, cit., p. 66, ap. 6.

51 Comisión de Derecho Internacional, Anuario de la CDI 2001, cit., pp. 39 y 40 (párrs. 3 y 4) y 49-51. Como referencia general del tema, véase Frouville, Olivier de, "Attribution of 
explotación minera no se realiza, en este caso, a través de una actividad gubernamental, son las autoridades territoriales quienes otorgaron la autorización para concretarla y quienes tienen el deber de verificar que se cumplan las normas vigentes, tanto internas de ese Estado como aquellas del derecho internacional..$^{52}$

Adicionalmente, la violación de las reglas en materia de prevención del daño transfronterizo es compleja de acreditar. Del principio general de derecho procesal que rige la carga probatoria se deriva que Argentina debería demostrar que Chile no controló a la empresa minera, o que no lo hizo de manera eficiente. Ahora bien, la existencia de un daño transfronterizo sensible es una prueba importante, aunque no concluyente, de la violación..$^{53}$

Esta lectura ampliada de la controversia permite ejemplificar las particularidades de los casos en materia de daño transfronterizo. Al mismo tiempo, se evidencia el enfoque pragmático que priorizan los Estados, los cuales están más interesados en obtener una solución satisfactoria en el corto plazo que en invocar conceptos teóricos del derecho internacional general frente a un tribunal internacional. ${ }^{54}$ Una vez más, es relevante recurrir a los comentarios de Brownlie respecto del decisorio Corfu Channel: "The judgment of the Court provides a valuable reminder of the need to avoid generalizing principles and simplistic polarities in the sphere of State responsibility". ${ }^{55}$

Conduct to the State: Private Individuals”, en Crawford, James et al. (eds.), The Law of International Responsibility, Oxford-Nueva York, Oxford University Press, 2010, pp. 257-280.

52 Conforme a Birnie et al.: "Even where an activity causing environmental harm is conducted by private parties, as in the Trail Smelter or Pulp Mills Cases, the issue remains one of the state's responsibility for prevention, cooperation, and notification, which cannot be avoided by surrendering the activity itself into private hands". Birnie, Patricia et al., op. cit., p. 214.

53 De acuerdo con el enfoque utilizado por la CDI en su Proyecto de Principios de 2006: "La responsabilidad causal puede aliviar la carga que, de lo contrario, deberían soportar las víctimas para probar la culpa del explotador, pero no elimina las dificultades que entraña el establecimiento del nexo causal necesario entre el daño y el origen de la actividad”. Comisión de Derecho Internacional, Anuario de la CDI 2006, cit., p. 88, párr. 16.

54 Conforme a Xue: "Between States, international liability is usually not a simple matter of recovery, but more often a process of the settlement of disputes, where not only the individual rights of the victims are involved, but national interests also must be accommodated". Xue, Hanqin, op. cit., p. 319.

55 Brownlie, Ian, op. cit., p. 16. 


\section{CONCLUSIONES}

En la primera parte de este trabajo fue expuesto el estado actual de los debates acerca de las reglas en materia de responsabilidad ex delicto y sine delicto. Asimismo, se destacó que, frente a un incidente por contaminación transfronteriza, en el que teóricamente podrían aplicarse los principios de la responsabilidad sine delicto, los Estados involucrados se enfrentan a un doble desafío: primero, un conjunto de reglas que aún no son reputadas como parte del derecho internacional general, y, segundo, una relativa complejidad en materia probatoria, que dificulta prever el éxito en un litigio jurisdiccional internacional.

En este sentido, la controversia entre Argentina y Chile por el vertedero en la Cordillera de los Andes resulta ilustrativa de los desafíos antes mencionados. Con el factor temporal en su contra, dado que los daños ambientales serían cada vez de más difícil mitigación, los Estados buscaron soluciones puntuales a través de negociaciones directas. Por ello, los acuerdos se concretaron mediante cuatro reuniones de un día de duración cada una, en un plazo total de cinco meses, y se formalizaron en el segundo mes posterior al último encuentro. Sin embargo, en el segundo convenio bilateral se dejó en claro que las posiciones jurídicas de las partes permanecían inalteradas, párrafo que habría sido incluido para preservar, fundamentalmente, la posición de Argentina. Esto implica que, a pesar de la búsqueda expeditiva de una solución, el Estado afectado se reservó el derecho de estudiar con más detenimiento la cuestión y, si fuera necesario, iniciar otro tipo de reclamación hacia Chile, por la violación de su soberanía y el daño ambiental provocado en su territorio.

Esta cláusula convencional evidencia que las reglas clásicas en materia de responsabilidad ex delicto podrían ser plenamente aplicables si así lo considera necesario Argentina, a pesar de que, desde una óptica teórica, el conflicto parece representar un evento de responsabilidad sine delicto, por hechos perjudiciales derivados de una actividad lícita.

De esta manera, la solución convenida para esta controversia se alejó de un esquema normativo rígido o de categorías estancas. Por el contrario, se adoptó un enfoque pragmático, que permite obtener una compensación para quienes hayan sufrido el daño concreto, así como una reparación a los intereses específicos del Estado afectado. 
VII. BIBLIOGRAFÍA

BARBERIS, Julio, El territorio del Estado y la soberanía territorial, Buenos Aires, Ábaco, 2003.

BARBOZA, Julio, "La responsabilidad sine delicto en el derecho internacional", Revista Jurídica de Buenos Aires, 2012.

BARBOZA, Julio, "Sine Delicto (Causal) Liability and Responsibility for Wrongful Acts in International Law", en INTERNATIONAL LAW COMMISSION, International Law on the Eve of the Twenty-First Century. Views from the International Law Commission, Nueva York, Naciones Unidas, 1997.

BARBOZA, Julio, The Environment, Risk and Liability in International Law, Leiden, Martinus Nijhoff, 2011.

BIRNIE, Patricia et al., International Law and the Environment, 3a. ed., Oxford University Press, 2009.

BOYLE, Alan E., "State Responsibility and International Liability for Injurious Consequences of Acts Not Prohibited by International Law: A Necessary Distinction?", The International and Comparative Law Quarterly, Cambridge, vol. 39, núm. 1, enero de 1990.

BROWNLIE, Ian, "State Responsibility and the International Court of Justice”, en Fitzmaurice, Malgosia y SArooshi, Dan (eds.), Issues of State Responsibility before International Judicial Institutions, Oxford, Hart Publishing, 2004.

Comisión de Derecho Internacional, Anuario de la CDI 1970, Nueva York, Naciones Unidas, 1972, vol. II.

Comisión de Derecho Internacional, Anuario de la CDI 1997, Nueva York-Ginebra, Naciones Unidas, 2002, vol. I.

Comisión de Derecho Internacional, Anuario de la CDI 2001, vol. II: Segunda parte, Nueva York-Ginebra, Naciones Unidas, 2007.

COMisión de Derecho InTERnaCiOnal, Anuario de la CDI 2006, vol. II: Segunda parte, Nueva York-Ginebra, Naciones Unidas, 2013.

CRAWFORD, James, State Responsibility:The General Part, Cambridge University Press, 2013.

CRAWFORD, James, "The International Court of Justice and the Law of State Responsibility", en TAms, Christian J. y SLOAN, James (eds.), The Develop- 
Esta revista forma parte del acervo de la Biblioteca Jurídica Virtual del Instituto de Investigaciones Jurídicas de la UNAM

ment of International Law by the International Court of Justice, Oxford University Press, 2013.

DAVID, Eric, "Primary and Secondary Rules", en CRAWFORD, James et al. (eds.), The Law of International Responsibility, Oxford-Nueva York, Oxford University Press, 2010.

DesCAmps, Hannes et al., International Documents on Environmental Liability, Heidelberg, Springer Netherlands, 2008.

DiEZ DE VELASCO, Manuel, Instituciones de derecho internacional público, Madrid, Tecnos, 2007.

DuPuY, Pierre-Marie, "The International Law of States Responsibility: Revolution or Evolution?", Michigan Journal of International Law, Míchigan, vol. 11, 1989.

Foster, Caroline, "The ILC Draft Principles on the Allocation of Loss in the Case of Transboundary Harm Arising out of Hazardous Activities: Privatizing Risk?", Review of European Community \& International Environmental Law, Nueva Jersey, vol. 14, núm. 3, 2005.

Frouville, Olivier de, "Attribution of Conduct to the State: Private Individuals", en CRAWFORD, James et al. (eds.), The Law of International Responsibility, Oxford-Nueva York, Oxford University Press, 2010.

GÓmEZ-Robledo VerduzCO, Alonso, Responsabilidad internacional por daños transfronterizos, 2a. ed., México, UNAM, 2014.

HANDL, Günther, "International Accountability for Transboundary Environmental Harm Revisited: What Role for «State Liability»?", Environmental Policy and Law, Ámsterdam, vol. 37, 2007.

HANDL, Günther, "State Liability for Accidental Transnational Environmental Damage by Private Persons", The American Journal of International Law, vol. 74, núm. 3, julio de 1980.

HANDL, Günther, "Territorial Sovereignty and the Problem of Transnational Pollution", The American Journal of International Law, vol. 69, núm. 1, enero de 1975.

LOIBL, Gerhard, "Environmental Law and Non-Compliance Procedures: Issues of State Responsibility", en FITZMAURICE, Malgosia y SAROOSHI, Dan (eds.), Issues of State Responsibility before International Judicial Institutions, Oxford, Hart Publishing, 2004.

MAGRAw, Daniel Barstow, "Transboundary Harm: The International Law Commission's Study of «International Liability»”, The American Journal of International Law, vol. 80, núm. 2, abril de 1986. 
Noguera Ramos, Gastón, “Caso «Pelambres»: usurpación, conflictos de límites y potencialidad de daño", Revista de Derecho Ambiental, Buenos Aires, núm. 53, 2018.

RaO, Pemmaraju Sreenivasa, "International Liability for Transboundary Harm”, Environmental Policy and Law, Ámsterdam, vol. 34, 2004.

Remiro Brotóns, Antonio, Derecho internacional, Valencia, Tirant Lo Blanch, 2007.

REY CARO, Ernesto J., "La responsabilidad internacional. Reflexiones sobre su evolución”, en REY CARO, Ernesto J. (dir.), Responsabilidad internacional, Córdoba, Academia Nacional de Derecho y Ciencias Sociales, 2009.

ReZeK, Francisco, Direito internacional público, 11 a. ed., São Paulo, Saraiva, 2008.

ROSAS, Allan, "Issues of State Liability for Transboundary Environmental Damage", Nordic Journal of International Law, Leiden, vol. 60, núm. 2, 1991.

Rosenne, Shabtai, The Perplexities of Modern International Law, Leiden, Martinus Nijhoff, 2004.

SANDS, Philippe, "Litigating Environmental Disputes: Courts, Tribunals and the Progressive Development of International Environmental Law", Environmental Policy and Law, Ámsterdam, vol. 37, 2007.

SCOVAZZI, Tullio, "Some Remarks on International Responsibility in the Field of Environmental Protection”, en RAGAZZI, Maurizio (ed.), International Responsibility Today. Essays in Memory of Oscar Schachter, Leiden, Koninklijke Brill NV, 2005.

SUCHARITKUL, Sompong, "State Responsibility and International Liability under International Law”, Loyola of Los Angeles International and Comparative Law Journal, Los Ángeles, vol. 18, 1996.

XUE, Hanqin, Transboundary Damage in International Law, Cambridge University Press, 2003.

YAMADA, Chusei, "Revisiting the International Law Commission's Draft Articles on State Responsibility", en RAGAZZI, Maurizio (ed.), International Responsibility Today. Essays in Memory of Oscar Schachter, Leiden, Koninklijke Brill NV, 2005. 